\title{
Promoção da aquisição de produtos da agricultura familiar para a alimentação escolar em Territórios da Cidadania de Minas Gerais e Espírito Santo
}

\author{
Promoting the purchase of family farm \\ products for school meals in Territories \\ of Citizenship of Minas Gerais and \\ Espírito Santo States, Brazil
}

\author{
Olívia Maria de Paula Alves BEZERRA ${ }^{1}$ \\ Élido BONOMO ${ }^{1}$ \\ Camilo Adalton Mariano da SILVA' \\ Margareth da Silva CORREA ${ }^{2}$ \\ Anelise Andrade de SOUZA ${ }^{3}$ \\ Peterson Cleber Teixeira dos SANTOS ${ }^{3}$ \\ Maria Luiza da SILVA ${ }^{4}$ \\ Marcelo Rodrigues de ALMEIDA ${ }^{3}$ \\ Karla Patrício CARVALHO ${ }^{3}$ \\ Luciana Araújo MARQUES 3 \\ Débora Maria Soares de SOUZA 3
}

\section{RE S U M O}

\section{Objetivo}

Contribuir para a promoção da aquisição de produtos da agricultura familiar para a alimentação escolar em Territórios da Cidadania do País.

\footnotetext{
1 Universidade Federal de Ouro Preto, Escola de Nutrição, Departamento de Nutrição Clínica e Social. Campus Morro do Cruzeiro, 35400-000, Ouro Preto, MG, Brasil. Correspondência para/Correspondence to: OMPA BEZERRA. E-mail: <ompab@yahoo.com.br>.

${ }^{2}$ Universidade Federal de Ouro Preto, Escola de Nutrição, Departamento de Alimentos. Ouro Preto, MG, Brasil.

3 Universidade Federal de Ouro Preto, Escola de Nutrição, Centro Colaborador em Alimentação e Nutrição do Escolar. Ouro

Preto, MG, Brasil.

${ }^{4}$ Ministério da Educação. Fundo Nacional do Desenvolvimento da Educação. Brasília, DF, Brasil.
} 
336 | OMPA BEZERRA et al.

\section{Métodos}

Foi realizado um estudo de intervenção com abordagem de articulação de redes intersetoriais presenciais, desenvolvidas a partir da atuação de equipes compostas por nutricionista, educador popular e engenheiro agrônomo ou técnico agrícola, que visitaram, por pelo menos dois momentos, todos os municípios pertencentes aos Territórios da Cidadania, visando articular redes intersetoriais presenciais capazes de desencadear processos locais de mobilização, organização e apoderamento de diferentes atores para a aquisição de produtos da agricultura familiar para a alimentação escolar nos municípios que compõem os referidos territórios.

\section{Resultados}

Os resultados mais expressivos foram: 14\% dos municípios visitados apresentaram melhora na organização da agricultura familiar; $14 \%$ dos gestores passaram a apoiar o processo de aquisição de alimentos segundo a Lei n 11.947/2009, e 27\% dos municípios que não possuíam nutricionista contrataram o profissional. Não foram considerados satisfatórios os resultados obtidos para situação do Conselho de Alimentação Escolar.

\section{Conclusão}

As ações desenvolvidas foram capazes de contribuir positivamente para o avanço da implementação da Lei Federal n 11.947/2009 nos Territórios de Cidadania abordados.

Termos de indexação: Agricultura familiar. Alimentação escolar. Articulação de redes. Territórios da Cidadania.

\section{A B S T R A C T}

\section{Objective}

This study hopes to promote the acquisition of family farm products for school meals in Brazil's Territories of Citizenship.

\section{Methods}

This intervention study coordinated intersectoral networks face-to-face. The networks consisted of teams composed of a dietician, popular educator, and agronomist or agronomy technician. These teams visited all municipalities within the Citizenship Territories at least twice to coordinate the said networks, capable of promoting local mobilization processes, organization, and empowerment of different actors to acquire family farm products for school meals in the said municipalities.

\section{Results}

The most expressive results were: $14 \%$ of the municipalities improved the family farm organization; $14 \%$ of the managers began to support the food acquisition process according to Law $n^{\circ} 11.947 / 2009$; and 27\% of the municipalities without a dietician hired one. The results were not considered satisfactory by the School Food Council.

\section{Conclusion}

The actions were capable of promoting the implementation of the Federal Law Lei Federal $n^{\circ} 11.947 / 2009$ in the study Territories of Citizenship.

Indexing terms: Family farm. School food. Network articulation. Territories of Citizenship.

\section{N T R O D U ÇÃ O}

O programa Territórios da Cidadania (TC) constitui estratégia governamental de desenvolvimento territorial cujos objetivos incluem promoção de desenvolvimento econômico e redução das desigualdades sociais por meio da universalização de programas básicos de cidadania1. Na proposta inicial, foram previstas 169 ações a serem desenvolvidas até 2010 nos 1851 municípios que compõem os 120 TC, os quais apresentam baixo dinamismo econômico e os menores Índices de Desenvolvimento Humano (IDH) do País ${ }^{2,3}$. A previsão de investimentos foi da ordem de $R \$ 27.393 .167 .127,25$, sendo executados $\mathrm{R} \$ 19.702 .775 .371,22^{3}$ até 31 de dezembro de 2010. 
Na Região Sudeste existem 16 TC, sendo 9 em Minas Gerais, 2 no Espírito Santo, 3 em São Paulo e 2 no Rio de Janeiro 4 . O Centro Colaborador em Alimentação e Nutrição do Escolar da Universidade Federal de Ouro Preto (CECANE/ UFOP) vem desenvolvendo ações em TC de Minas Gerais e Espírito Santo, buscando contribuir para o fornecimento dos produtos da Agricultura Familiar (AF) para a alimentação escolar, com base na Lei Federal $n^{\circ} 11.947 / 2009^{5}$ e na Resolução CD/FNDE $n^{\circ} 38 / 2009^{6}$.

Este artigo é oriundo do produto "Qualificação de atores na perspectiva da implementação da Lei 11.947 em Territórios de Cidadania"5, desenvolvido pelo CECANE/UFOP mediante financiamento do Fundo Nacional de Desenvolvimento da Educação (FNDE), no ano de 2011. Nele são relatados os resultados obtidos na promoção da aquisição dos produtos da agricultura familiar para a alimentação escolar nos TC Norte (ES), Alto, Médio e Baixo Jequitinhonha (MG). Pretende-se, assim, contribuir para o desenvolvimento de ações de promoção da aquisição de produtos da agricultura familiar em TC do País.

\section{M É T O D O S}

A concepção de território empregada neste artigo seguiu a linha defendida por Cuzella et al.7: a de que o território não é apenas uma realidade geográfica ou física, mas uma realidade humana, social, cultural e histórica resultante de uma construção social realizada por um sistema local de atores. É, portanto, um espaço onde se estabelecem relações com outros atores sociais, instituições e territórios ${ }^{8}$.

Considerando que a própria concepção de desenvolvimento territorial: "Deveria incorporar como uma de suas prerrogativas a ideia de que não bastam melhorias produtivas, econômicas e sociais se estas não forem acompanhadas de uma aprendizagem que permita que as pessoas sejam capacitadas para, ao final, ser incumbidas de assumir a responsabilidade pela sua conti- nuidade..." (p.19) ${ }^{9}$, buscou-se a abordagem metodológica de articulação de redes intersetoriais presenciais $^{10}$ visando ao desencadeamento de processos locais de mobilização, organização e apoderamento de diferentes atores envolvidos na aquisição de produtos da agricultura familiar para a alimentação escolar. Partiu-se do princípio de que a organização de redes intersetoriais pressupõe a construção coletiva do espaço de organização dos sujeitos mobilizados por objetivos elaborados e apropriados coletivamente para a promoção de uma nova realidade social ${ }^{11,12}$.

As ações foram desenvolvidas nos TC Norte (ES), Alto, Médio e Baixo Jequitinhonha (MG). Inicialmente, a proposta foi apresentada aos órgãos diretivos dos TC, o que ocorreu no Seminário Estadual "Comercialização Institucional Programa de Aquisição de Alimento net (PAAnet) e Programa Nacional de Alimentação Escolar (PNAE)", realizado em Belo Horizonte em 2010. Posteriormente, solicitou-se sua discussão em reuniões dos colegiados territoriais para que fosse aprovada, quando recebeu sugestões diversas que a enriqueceram. Desse modo, as ações desenvolvidas foram construídas em parceria com os colegiados territoriais, sendo organizadas a partir da composição de quatro equipes formadas por um nutricionista, um educador popular e um engenheiro agrônomo ou técnico agrícola, os quais deveriam residir ou ter trabalhado no TC; conhecer bem os municípios do TC; e ter experiência em articulação de redes sociais e em educação popular. Sempre que possível, o articulador territorial fez parte da equipe.

As equipes passaram por treinamento no CECANE/UFOP para dar maior conhecimento sobre o PNAE, Lei no 11.947/20095 e Resolução FNDE $n^{\circ} 38 / 2009^{6}$. A seguir, foi realizada oficina de trabalho nos TC, quando eram apresentados os fundamentos do PNAE, os treze passos para a aquisição dos produtos da agricultura familiar para a alimentação escolar e discutidas as características da agricultura familiar local. Participaram das oficinas Componentes do Colegiado Territorial 
e diversos atores locais relacionados ao PNAE e à agricultura familiar.

Só então foram iniciados os trabalhos de campo - cuja duração, em cada território, foi de três meses -, definidos em função da possibilidade de se cumprir, nesse período, um ciclo completo de compras segundo a Lei $n^{0} 11.947 / 2009^{5}$ e das possibilidades legais de contratação das equipes.

O trabalho das equipes consistia em visitar todos os municípios do TC em pelo menos dois momentos, definindo a frequência e o intervalo entre as visitas segundo a necessidade identificada. Durante essas visitas, realizavam-se reuniões com diferentes atores para discutir aspectos da execução do PNAE e da implementação da Lei $n^{\circ}$ $11.947 / 2009^{5}$, promovendo as articulações necessárias para que os entraves fossem superados. Durante a primeira visita, eram pactuadas ações a serem desenvolvidas, e nas visitas subsequentes, realizavam-se novas reuniões para discutir as ações implementadas e desenvolver novas articulações.

Cada equipe elaborou um diário de campo, no qual se registravam informações sobre as reuniões realizadas. Elaboraram-se, também, relatórios mensais e finais, repassados ao CECANE/UFOP para registro e acompanhamento das atividades. As variáveis estudadas incluíram a situação do Conselho de Alimentação Escolar (CAE), a contratação de nutricionistas, a organização dos agricultores familiares, o acesso ao Programa de Aquisição de Alimentos (PAA), a gestão municipal em relação ao apoio à compra da agricultura familiar e o processo de compra em todos os seus aspectos. As informações obtidas foram sistematizadas em planilha Exce/ versão $8.0^{13} \mathrm{e}$ analisadas no Programa Epi Info, versão 3.5.2 $2^{14}$.

Ao final dos trabalhos de campo, foram realizadas novas reuniões entre o CECANE/UFOP, as equipes e os colegiados territoriais, visando avaliar os resultados das ações desenvolvidas e propor novos encaminhamentos. Os municípios dos quatro TC permanecem acompanhados à distância pelo CECANE/UFOP, que deve prestar apoio técnico por meio de participação em reuniões, palestras e eventos, com o intuito de esclarecer as principais dúvidas sobre o PNAE e fortalecer a articulação dos atores locais envolvidos no Programa.

\section{RESULTADOS E DISCUSSÃ O}

Foram realizadas duas visitas aos 17 municípios do TC Norte (ES). No TC Alto Jequitinhonha, foram realizadas duas visitas a 8 municípios $(34,8 \%)$ e três visitas a 15 municípios $(65,2 \%)$. No TC Médio Jequitinhonha, foram realizadas duas visitas aos 18 municípios, porém em 2 deles, Berilo e Novo Cruzeiro, não foi possível realizar a reunião da segunda visita devido à baixa mobilização dos atores, levando à insuficiência de participantes e à consequente exclusão da análise. No TC Baixo Jequitinhonha, foram realizadas duas visitas a 10 municípios $(62,5 \%)$ e 3 visitas a 6 municípios (37,5\%), devido à constatação de pouca mobilização dos atores para efetivação da compra segundo a Lei $n^{\circ} 11.947 / 2009$.

Os desafios de implementar essa Lei no País adquirem dimensões ainda maiores nos TC, cujos municípios apresentam os mais baixos IDH do País ${ }^{3}$. Por isso, pretendeu-se abranger todos os municípios dos TC abordados. Os resultados das ações realizadas nos quatro TC estão descritos na Tabela 1.

Em relação à situação dos $C A E$, os resultados obtidos não foram considerados satisfatórios, sugerindo incapacidade das equipes em contribuir positivamente para a melhoria do controle social, especialmente nos TC Alto e Médio Jequitinhonha. Verificou-se que, embora a situação do CAE tenha se mantido adequada em $75 \%$ dos municípios, houve redução de aproximadamente 20\% no número de municípios com CAE válido e aumento do número de municípios com CAE diligenciado e em análise (Tabela 1), o que indica uma possível limitação técnica ou metodológica das ações desenvolvidas, possivelmente relacionadas ao tempo de permanência das equipes nos territórios, que parece ter sido insuficiente para promover os necessários avanços no controle social. Entretanto, há que se considerar que a 
Tabela 1. Resultados das ações realizadas pelo CECANE/UFOP nos TC Norte (ES), Alto, Médio e Baixo Jequitinhonha (MG).

\begin{tabular}{|c|c|c|c|c|c|c|c|c|c|c|c|c|c|c|c|c|c|c|c|c|}
\hline & \multicolumn{4}{|c|}{$\begin{array}{l}\text { Norte - ES } \\
(n=17)\end{array}$} & \multicolumn{4}{|c|}{$\begin{array}{l}\text { Alto Jequitinhonha } \\
\qquad(\mathrm{n}=23)\end{array}$} & \multicolumn{4}{|c|}{$\begin{array}{l}\text { Médio Jequitinhonha } \\
\qquad(\mathrm{n}=16)\end{array}$} & \multicolumn{4}{|c|}{$\begin{array}{l}\text { Baixo Jequitinhonha } \\
\qquad(\mathrm{n}=16)\end{array}$} & \multicolumn{4}{|c|}{$\begin{array}{c}\text { Total } \\
(\mathrm{n}=72)\end{array}$} \\
\hline & \multicolumn{2}{|c|}{$\begin{array}{l}\text { Primeira } \\
\text { visita* }\end{array}$} & \multicolumn{2}{|c|}{$\begin{array}{l}\text { Última } \\
\text { visita* }\end{array}$} & \multicolumn{2}{|c|}{$\begin{array}{l}\text { Primeira } \\
\text { visita* }^{*}\end{array}$} & \multicolumn{2}{|c|}{$\begin{array}{l}\text { Última } \\
\text { visita* }\end{array}$} & \multicolumn{2}{|c|}{$\begin{array}{l}\text { Primeira } \\
\text { visita* }^{*}\end{array}$} & \multicolumn{2}{|c|}{$\begin{array}{l}\text { Última } \\
\text { visita* }\end{array}$} & \multicolumn{2}{|c|}{$\begin{array}{l}\text { Primeira } \\
\text { visita* }\end{array}$} & \multicolumn{2}{|c|}{$\begin{array}{l}\text { Última } \\
\text { visita* }\end{array}$} & \multicolumn{2}{|c|}{$\begin{array}{l}\text { Primeira } \\
\text { visita* }\end{array}$} & \multicolumn{2}{|c|}{$\begin{array}{l}\text { Última } \\
\text { visita* }\end{array}$} \\
\hline & $n$ & $\%$ & $n$ & $\%$ & $n$ & $\%$ & $n$ & $\%$ & $\mathrm{n}$ & $\%$ & $n$ & $\%$ & $n$ & $\%$ & $\mathrm{n}$ & $\%$ & $\mathrm{n}$ & $\%$ & $\mathrm{n}$ & $\%$ \\
\hline \multicolumn{21}{|l|}{$C A E$} \\
\hline Diligenciado & 2 & 11,8 & 2 & 11,8 & - & - & 7 & 31,5 & - & - & 3 & 18,7 & - & - & - & - & 2 & 2,8 & 12 & 16,0 \\
\hline Em análise & - & - & - & - & - & - & - & - & - & - & 4 & 25,0 & - & - & - & - & - & - & 4 & 6,0 \\
\hline Vencido & - & - & - & - & - & - & - & - & - & - & - & - & 2 & 12,5 & 2 & 12,5 & 2 & 2,8 & 2 & 3,0 \\
\hline Não tem nutricionista & 1 & 5,9 & 1 & 5,9 & 5 & 21,7 & 3 & 13,0 & 2 & 12,5 & 3 & 18,8 & 7 & 43,8 & 5 & 31,2 & 15 & 20,8 & 12 & 16,7 \\
\hline \multicolumn{21}{|l|}{ Agricultura familiar } \\
\hline Organizada & 17 & 100,0 & 17 & 100,0 & 7 & 30,5 & 17 & 74,0 & 9 & 56,2 & 5 & 31,2 & 9 & 56,3 & 13 & 81,3 & 42 & 58,3 & 52 & 72,2 \\
\hline Não organizada & - & - & - & - & 16 & 69,5 & 6 & 26,0 & 7 & 43,8 & 11 & 68,8 & 7 & 43,8 & 3 & 18,8 & 30 & 41,7 & 20 & 27,8 \\
\hline \multicolumn{21}{|l|}{ PAA } \\
\hline Sim & 14 & 82,3 & 14 & 82,3 & - & - & - & - & 10 & 62,5 & 10 & 62,5 & 3 & 18,8 & 3 & 18,8 & 27 & 37,5 & 27 & 37,5 \\
\hline \multicolumn{21}{|c|}{$\begin{array}{l}\text { Situação da gestão municipal em relação à } \\
\text { compra da agricultura familiar }\end{array}$} \\
\hline $\begin{array}{l}\text { Gestor não apoia a } \\
\text { compra }\end{array}$ & 1 & 5,8 & - & - & 3 & 13,0 & 3 & 13,0 & 3 & 18,8 & 2 & 12,5 & 8 & 50,0 & - & - & 15 & 20,8 & 5 & 7,0 \\
\hline \multicolumn{21}{|c|}{$\begin{array}{l}\text { Situação do município em relação à compra da } \\
\text { agricultura familiar }^{2}\end{array}$} \\
\hline $\begin{array}{l}\text { Ainda não iniciou } \\
\text { discussão }\end{array}$ & - & - & - & - & 2 & 8,7 & - & - & 6 & 37,5 & 3 & 18,8 & 5 & 31,3 & 2 & 12,5 & 13 & 18,0 & 5 & 7,0 \\
\hline $\begin{array}{l}\text { Em articulação com } \\
\text { agricultura familiar }\end{array}$ & 2 & 11,8 & 2 & 11,8 & 1 & 4,3 & 1 & 4,3 & 32 & 12,5 & 2 & 12,5 & 7 & 43,8 & - & - & 12 & 16,7 & 5 & 7,0 \\
\hline $\begin{array}{l}\text { Elaborando chamada } \\
\text { pública }\end{array}$ & 1 & 5,8 & 2 & 11,8 & 1 & 4,3 & 2 & 8,7 & - & - & 7 & 43,8 & - & - & 3 & 18,8 & 2 & 2,8 & 14 & 19,4 \\
\hline $\begin{array}{l}\text { Chamada pública } \\
\text { publicada }\end{array}$ & 2 & 11,8 & 1 & 5,8 & 1 & 4,3 & 1 & 4,3 & - & - & 2 & 12,5 & 2 & 12,5 & 4 & 25,0 & 5 & 6,9 & 8 & 11,0 \\
\hline $\begin{array}{l}\text { Em análise do projeto de } \\
\text { venda }\end{array}$ & - & - & 1 & 5,8 & - & - & - & - & - & - & - & - & - & - & - & - & - & - & 1 & 1,4 \\
\hline Contratos já assinados & - & - & 1 & 5,8 & - & - & - & - & - & - & 1 & 6,25 & - & - & - & - & - & - & 2 & 2,8 \\
\hline
\end{tabular}

Nota: ${ }^{1}$ Informação não foi coletada durante as visitas. ${ }^{2}$ Cinco municípios do Norte (ES) e 11 municípios do Médio Jequitinhonha (MG) iniciaram novo processo de compras durante os trabalhos, sendo contabilizados em uma das fases do processo na segunda visita.

PAA: Programa de Aquisição de Alimento. *Número de municípios. 
fonte de obtenção do status do CAE é o sistema online do FNDE, intitulado "Espelho do CAE", e que os motivos de diligência dos conselhos podem estar ligados a outros fatores não relacionados à atuação da equipe, como, por exemplo, ausência de ata de eleição de um dos segmentos do $C A E^{15}$. De fato, promover um controle social efetivo parece ser um dos principais desafios do PNAE ${ }^{16}$. Os resultados encontrados sugerem a necessidade de ações mais incisivas das equipes junto aos CAE durante a sua permanência nos TC, assim como de revisão da metodologia utilizada e a inclusão de outras ações de formação continuada para conselheiros do CAE.

Verificou-se efeito positivo na contratação de nutricionistas nos TC Alto e Baixo Jequitinhonha, pois, em $27 \%$ dos municípios que não possuíam esse profissional, foi promovida a sua contratação, fato possivelmente relacionado à capacidade das equipes de sensibilizar os gestores para a necessidade e a importância desse profissional para o planejamento e a execução do PNAE. No entanto, em aproximadamente $17 \%$ dos municípios, não foi realizada a contratação desse profissional durante a realização dos trabalhos, sendo preocupante a persistência dessa situação nos quatro territórios, já que a contratação do nutricionista é condição básica para a execução do programa (Tabela 1). Alguns estudos vêm apontando a ausência do nutricionista como uma das principais dificuldades enfrentadas pelo PNAE ${ }^{17,18}$. Os resultados encontrados indicam a necessidade de ações mais incisivas das equipes junto aos gestores e CAE municipais a fim de regularizar a situação perante o FNDE. No entanto, há que se considerar que, embora alguns municípios não tenham contratado o profissional durante o período de permanência da equipe, pode ter havido sensibilização dos gestores para posterior contratação.

Outra atuação positiva das equipes pode ser constatada na organização dos agricultores familiares em associações e cooperativas para a comercialização de seus produtos para a alimentação escolar, já que 14\% dos municípios visitados apresentaram melhora na organização da agricultura familiar. Em alguns municípios, houve progresso importante na organização dos agricultores familiares, com destaque para os TC Alto e Baixo Jequitinhonha (Tabela 1), favorecendo sobremaneira a aquisição dos produtos da agricultura familiar para o PNAE, já que a organização social em associações e cooperativas facilita o acesso às chamadas públicas e aos mercados institucionais ${ }^{19}$.

Apenas no TC Médio Jequitinhonha foi constatada redução do número de municípios onde a agricultura familiar já se encontrava organizada, no entanto esse achado pode ser atribuído a um possível viés de informação, já que, na primeira visita, a informação foi dada por agricultores familiares, e, na segunda, pelos gestores (Tabela 1). Assim, afirmações sobre o grau de organização dos agricultores familiares nesse território constitui uma limitação deste artigo.

Ainda permanece inadequada a organização da agricultura familiar em aproximadamente $28 \%$ dos municípios (Tabela 1), indicando a necessidade de ações mais afirmativas ou maior tempo de atuação das equipes junto aos agricultores familiares e suas entidades de classe, de modo a contribuir efetivamente para a formação de associações e cooperativas de agricultores familiares e empreendedores familiares rurais para a comercialização de seus produtos para o PNAE ${ }^{19}$. É sabido que a formação de uma base organizativa sólida gera melhores condições para a execução do PNAE e para a implementação da Lei $n^{\circ} 11.947 / 2009^{5}$.

Com relação ao PAA, foi constatada boa adesão dos municípios nos TC Norte - ES e Médio Jequitinhonha. No entanto, no Baixo Jequitinhonha, a adesão ao programa mostrou-se baixa. Já no TC Alto Jequitinhonha e em alguns municípios do Médio Jequitinhonha, a informação não foi coletada, e os autores optaram por não recorrer aos registros oficiais para não alterar a metodologia adotada (Tabela 1). Ressalta-se que essas adesões ao PAA não guardam relação direta com a atuação das equipes nos territórios por não terem sido objeto de sua intervenção, mas constituem importantes indicadores do nível de organização da agricultura familiar local e da capa- 
cidade do município em implementar a Lei $\mathrm{n}^{\circ}$ $11.947 / 2009^{5}$.

Já em relação ao apoio do gestor, verificou-se que as ações desenvolvidas foram capazes de promover aumento substancial no número de municípios onde os gestores apoiavam a compra, passando de $79 \%$ para $93 \%$, o que sugere um resultado bastante positivo do trabalho das equipes e contribui favoravelmente para o sucesso da compra. Os avanços nesse aspecto foram mais acentuados no TC Baixo Jequitinhonha, mas também ocorreram nos TC - Norte (ES) e Médio Jequitinhonha, nos quais já se verificava apoio expressivo dos gestores antes do início dos trabalhos. A situação se manteve inalterada apenas no TC Alto Jequitinhonha, onde o apoio dos gestores também já ocorria na maioria dos municípios (Tabela 1). O comprometimento dos gestores constitui uma das principais condições para o êxito das políticas públicas, sendo essa condição essencial para a implementação da Lei $n^{\circ} 11.947 / 2009^{5}$. Entretanto, naqueles municípios em que não foi verificado tal apoio, torna-se necessário o desenvolvimento de novas ações de sensibilização por parte do CECANE/UFOP e dos órgãos governamentais, buscando ampliar o acesso dos agricultores familiares ao PNAE.

Em relação à compra da agricultura familiar propriamente dita, verificou-se efeito bastante positivo das ações das equipes nos quatro TC, já que 8 municípios que não haviam iniciado as discussões conseguiram avanços importantes nas etapas do processo durante a permanência das equipes nos territórios, e, em alguns deles, já se iniciava um segundo ciclo de compras a partir de nova chamada pública. Em 93\% dos 72 municípios visitados, constataram-se avanços no processo de compra ou a situação se manteve favorável, e, ao término dos trabalhos, mais da metade desses municípios já se encontrava em fase de recebimento dos produtos da agricultura familiar, e cerca de $26 \%$ deles conseguiram iniciar as articulações com a agricultura familiar ou se encontravam em processo de elaboração da chamada pública (Tabela 1). Destaca-se a necessidade de ações mais efetivas naqueles municípios onde a compra não foi iniciada durante os trabalhos das equipes, especialmente nos TC Médio e Baixo Jequitinhonha, onde ainda havia municípios que não iniciaram a discussão do processo de compra segundo a Lei $n^{\circ}$ 11.947/20095.

Ressalta-se que não foi localizada pelos autores a bibliografia que aborde a aquisição de produtos da agricultura familiar para o PNAE em TC, o que impediu a comparação dos resultados com achados de outros autores.

\section{O N CLUSÃ O}

A partir dos resultados obtidos nos quatro TC, constatou-se que as ações desenvolvidas foram capazes de contribuir positivamente para o avanço do processo de aquisição dos produtos da agricultura familiar para a alimentação escolar nos quatro territórios de cidadania. Os resultados mais expressivos ocorreram na organização dos agricultores familiares locais, na contratação de nutricionistas, no apoio dos gestores e na situação do município em relação à compra da agricultura familiar segundo a Lei no 11.947/20095.

No entanto, os resultados relativos à atuação do CAE não foram considerados satisfatórios, sugerindo uma possível limitação do método empregado ou da atuação das equipes. Evidencia-se, dessa forma, a necessidade de ações mais efetivas por parte do CECANE/UFOP e dos órgãos governamentais, capazes de efetivamente garantir a execução do controle social nos TC.

Destaca-se a importância da parceria com os colegiados e outras instâncias de gestão territorial na construção conjunta de propostas para as políticas públicas e no desenvolvimento das ações em nível municipal e territorial. Espera-se que os resultados aqui relatados possam efetivamente contribuir de forma positiva para o desenvolvimento de ações voltadas para a implementação da Lei $n^{\circ} 11.947^{5}$ nos TC brasileiros, a partir do aperfeiçoamento do método proposto e de seu ajuste à realidade e às particularidades de cada território. 


\section{A GRADECIMENTOS}

Ao Fundo Nacional de Desenvolvimento da Educação pelo apoio técnico e financeiro às atividades desenvolvidas nos Territórios de Cidadania.

\section{OLABORADORES}

OMPA BEZERRA planejou o estudo, conduziu os trabalhos de campo, as análises e a elaboração do artigo. E BONOMO, CAM SILVA, MS CORREA, colaboraram na elaboração do estudo, na realização dos trabalhos de campo, nas análises e na elaboração do artigo. AA SOUZA, PCT SANTOS, MR ALMEIDA, KP CARVALHO, LA MARQUES, DMS SOUZA colaboraram na realização dos trabalhos de campo, na elaboração das planilhas, nas análises e na elaboração do artigo. ML SILVA colaborou na elaboração do estudo, na realização dos trabalhos de campo e na elaboração do artigo.

\section{REFERÊ NCIAS}

1. Empresa Brasileira de Pesquisa Agropecuária. Proposta do Ministério do Desenvolvimento Agrário para redução da desigualdade social no meio rural brasileiro. Pelotas: Embrapa. [acesso 2011 out 18]. Disponível em: <http://www.cpact.embrapa.br/ forum>.

2. Brasil. Ministério do Desenvolvimento Agrário. Portal Notícias. Brasília: MDA; [s.d.]. [acesso 2011 out 20]. Disponível em: <http://www.mda.gov.br/ portal/noticias/item?item_id=3577820 $>$.

3. Portal Brasil. Territórios da cidadania. Brasília: Portal Brasil; [s.d.]. [acesso 2012 jul 24]. Disponível em: <http://www.territoriosdacidadania.gov.br/dotlrn/ clubs/territriosrurais/xowiki/portlets/territorios/ pages/historico_do_programa?sdt_code=>.

4. Ministério do Desenvolvimento Agrário. Brasil. Sistema de Informações Territoriais. Brasília: MDA; c2008 [acesso 2011 set 25]. Disponível em: <http:// sit.mda.gov.br/territorio. php?menu=cidadania \&base $=2>$.

5. Brasil. Lei $n^{\circ} 11.947$, de 16 de junho de 2009. Dispõe sobre o atendimento da alimentação escolar e do Programa Dinheiro Direto na Escola aos alunos da educação básica. Diário Oficial da União. 2009 17 jun 2009; p.2-4.

6. Brasil. Resolução/CD/FNDE $n^{\circ} 38$, de 16 de julho de 2009. Dispõe sobre o atendimento da alimentação escolar aos alunos da educação básica no Programa Nacional de Alimentação Escolar - PNAE. Diário Oficial da União. 200917 jul; Seção 1.
7. Cazella AA, Bonnal P, Maluf RS. Multifuncionalidade da agricultura familiar no Brasil e o enfoque da pesquisa. In: Cazella AA, Bonnal P, Maluf RS, Organizadores. Agricultura familiar: multifuncionalidade e desenvolvimento territorial no Brasil. Rio de Janeiro: Mauad X; 2009.

8. Saldanha JC, Antongiovanni L, Scarim PC. Diálogos entre a multifuncionalidade da agricultura familiar e os projetos coletivos de educação do campo e da agroecologia no Norte do Espírito Santo. In: Cazella AA, Bonnal P, Maluf RS, Organizadores. Agricultura familiar: multifuncionalidade e desenvolvimento territorial no Brasil. Rio de Janeiro: Mauad X; 2009.

9. Schneider S, Tartaruga IGP. Território e abordagem territorial das referências cognitivas aos aportes aplicados à análise dos processos sociais rurais. Rev Ciênc Sociais Econômicas. 2004; 23(1):19.

10. Castells M. A sociedade em rede. São Paulo: Paz e Terra; 1999.

11. Olivierie L. A importância histórico-social das redes. In: Manual de redes sociais e tecnologia. São Paulo: Conectas; 2002.

12. Junqueira LAP. A gestão intersetorial das políticas sociais e o terceiro setor. Saúde Soc. 2004; 13(1):25-36.

13. Microsoft Excel. Versão 8.0. Seatlle: Microsoft; 1997.

14. Epi Info. Versão 3.5.2. Atlanta: Centers of Disease Control; 2010.

15. Brasil. Fundo Nacional de Desenvolvimento da Educação. Consulta Espelho do CAE. Brasília: FNDE; [s.d.]. [acesso 2012 jul 31]. Disponível em: <https:// www.fnde.gov.br/pnaeweb/publico/consulta EspelhoCae.do>.

16. Triches RM, Schneider S. Alimentação escolar e agricultura familiar: reconectando o consumo à produção. Saúde Soc. 2010; 19(4):933-45.

17. Belik W, Chaim NA. O programa nacional de alimentação escolar e a gestão municipal: eficiência, administrativa, controle social e desenvolvimento local. Rev Nutr. 2009; 22(5):595-607. doi: 10.1590/ S1415-52732009000500001.

18. Chaves LG, Mendes PNR, Brito RR, Botelho RBA. O Programa Nacional de Alimentação Escolar como promotor de hábitos alimentares regionais. Rev Nutr. 2009; 22(6):857-66. doi: 10.1590/S1415-527 32009000600007.

19. Becker C, Andersson FS, Altemburg SGN, Costa LC. Novas concertações sociais e antigos entraves "burocráticos": a produção de base familiar e ecológica frente aos desafios de acesso ao PNAE. Cad Agroecol. 2011; 6(2).

Recebido em: 22/11/2011 Versão final em: 23/4/2012 Aprovado em: 15/5/2012 